\title{
AVALIAÇÃo do EFEITO do POLIELETRÓlito ANIÔNICO NA CLARIFICAÇÃo DO EXTRATO AgUOSO DE Stevia rebaudiana ${ }^{1}$
}

\author{
Élcio J. BUNHAK ${ }^{2 *}$, Elisabete S. MENDES ${ }^{2}$, Nehemias C. PEREIRA², Sílvio C. COSTA²
}

\begin{abstract}
RESUMO
A clarificação é uma etapa relevante no processo de obtenção de adoçantes a base de esteviosídeo. Neste trabalho, utilizou- se o polieletrólito aniônico (BETZDEARBORN $\left.{ }^{\mathrm{TM}} \mathrm{F} 11\right)$ na decantação da suspensão do extrato cru, que possui cor escura, e avaliou-se sua aplicabilidade. O extrato utilizado era obtido a partir de plantas selecionadas e modificadas geneticamente, no Núcleo de Estudos em Produtos Naturais (NEPRON) no Universidade Estadual de Maringá (UEM), o qual mantém uma linha de pesquisa denominada Projeto Stevia e planta piloto em parceria com a iniciativa privada. Os ensaios foram realizados em aparelho Jar Test divididos em duas etapas, que se distinguiam na dosagem do polímero e na utilização do sobrenadante ou da suspensão total após tratamento com óxido de cálcio, o qual realizava o ajuste do pH. Realizou-se leituras de absorbâncias em 420 (cor) e 670†m (turbidez) e, fez-se cálculos de percentagem de despigmentação e de velocidade de decantação. Os melhores resultados na etapa 1, onde se aplicou baixas quantida-

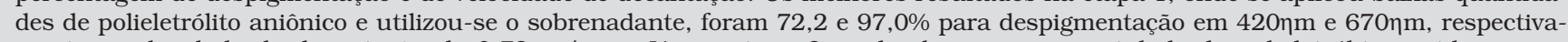
mente e velocidade de decantação de $0,72 \mathrm{~cm} / \mathrm{min}$. Já, na etapa 2 , onde elevou-se a quantidade do polieletrólito e utilizou-se a suspensão total, os melhores resultados obtidos foram de 70,5\% de despigmentação em $420 \eta \mathrm{m}, 85,6 \%$ de despigmentação em $670 \eta \mathrm{m}$ e velocidade de decantação de $0,71 \mathrm{~cm} / \mathrm{min}$.

Palavras-chave: clarificação; esteviosídeo; polieletrólito aniônico; percentagem de despigmentação.
\end{abstract}

\section{SUMMARY}

EVALUATION OF ANIONIC POLYELECTROLYTE EFFECT IN THE CLARIFICATION OF THE STEVIA REBAUDIANA AQUEOUS EXTRACT. The clarification is an important stage in the process of sweeteners for obtaining the stevioside. In this work, it was used the anionic polyelectrolyte (BETZDEARBORN $\left.{ }^{\mathrm{TM}} \mathrm{F} 11\right)$ for decantation of the raw extract suspension, which possesses dark color, and it was evaluated its sweats application. The used extract was obtained in first stage from selected plants and modified genetically, in the Nucleus of Studies in Natural Products of the Universidade Estadual de Maringá (UEM), which maintains a line of denominated research Project Stevia and its plant pilot works in partnership with the private initiative. The rehearsals were accomplished in apparel Jar Test divided in two stages, either distinguished in dosage of the polymer and in the use of the supernatant or of the total suspension after treatment with calcium oxide, which accomplished the adjustment of the pH. Absorbances were read in 420 (color) and $670 \eta m$ (turbidity) and, it was calculated the color removal percentage and the decantation speed. The best results in the stage 1 was when it was applied low amounts of anionic polyelectrolyte and the use of supernatant was 72.2 and $97.0 \%$ for color removal in $420 \eta \mathrm{m}$ and $670 \eta \mathrm{m}$ and the decantation speed was of $0.72 \mathrm{~cm} / \mathrm{min}$. At stage 2 , when it was used a risen amount of the polyelectrolyte and the total suspension, the best results obtained for color removal were of $70.5 \%$ in $420 \eta \mathrm{m}$, and $85.6 \%$ in $670 \eta \mathrm{m}$ and decantation speed was of $0.71 \mathrm{~cm} / \mathrm{min}$. Keywords: clarification; stevioside; anionic polyelectrolyte; color removal percentage.

\section{1 - INTRODUÇÃO}

Atualmente, no Brasil e em outros países, existe um grande interesse na pesquisa, plantio, industrialização, comercialização de Stevia rebaudiana (Bert.) Bertoni, isto devido aos seus princípios edulcorantes. Estes apresentam a possibilidade de seu uso em larga escala em alimentos, bebidas e medicamentos. Estes princípios edulcorantes contém os glicosídios que são não-tóxicos, não-mutagênicos e de baixo valor calórico. Podendo ser utilizados com sucesso como alternativas do açúcar tradicional para pacientes que sofrem de diabetes e outras doenças relacionadas ao metabolismo de carboidratos [1]. As folhas de Stevia rebaudiana (Bert.) Bertoni contêm uma mistura complexa de diterpenos labdanos, triterpenos, estigmasterol, taninos, óleos voláteis e oito glicosídios diterpenos adoçantes, que são: esteviosídeo, rebaudiosídeos A, B, C, D, E e dulcosideos A e B [6].

\footnotetext{
Recebido para publicação em 07/01/2003. Aceito para publicação em 12/07/2004 (001052).

2. Departamento de Engenharia Química, Universidade Estadual de Maringá; Av. Colombo, 5790; CEP: 87020-900-MARINGÁ-PR; Brasil. Fone:Oxx-44-261-4323; Fax:Oxx-44-261-3440. E-mail: elciojb@deq.uem.br * A quem a correspondência deve ser enviada.
}

Destes o rebaudiosídeo A é o mais doce, o mais estável e menos amargo que o esteviosídeo. O rebaudiosídeo E é tão doce quanto o esteviosídeo, e o rebaudiosídeo D quanto o rebaudiosídeo A, enquanto que os outros glicosídios são menos doces que o esteviosídeo [7].

As substâncias mais abundantes nas folhas da estévia são o esteviosídeo e os rebaudiosídeos, sendo a maior percentagem do esteviosídeo. Este glicosídio apresenta sabor residual desagradável, amargo e adstringente, que se percebe após o sabor doce, e tem ainda baixa solubilidade em água, o que dificulta sua adição nas formulações. Tudo isto limita sua utilização e tem motivado os pesquisadores a buscarem um melhoramento genético nas plantas de estévia, no que se relaciona a aumentar a quantidade do rebaudiosídeo A em relação ao esteviosídeo e também à elevação da quantidade de adoçantes totais por unidade de massa foliar [4]. A UEM através do NEPRON, já produz plantas modificadas de estévia, com características melhoradas conforme demonstrado na Tabela 1.

O extrato da estévia apresenta cor escura devido a grande quantidade de material orgânico e inorgânico em suspensão, que devem ser removidos para facilitar e otimizar as etapas subseqüentes do processo de purificação e refino. 
TABELA 1. Teores de edulcorantes em folhas de estévia e Stevia rebaudiana (Bert.) Bertoni

\begin{tabular}{ccc}
\hline Composição & Estévia Comum (\%) & Estévia UEM 320 (\%) \\
\hline Esteviosídeo & 9,1 & 6,5 \\
Rebaudiosídeo C & 1,2 & 1,3 \\
RebaudiosídeoA & 4,5 & 9,8 \\
Adoçantes totais & 14,8 & 17,6 \\
Razão Rebaudiosídeo A /Esteviosídeo & 0,5 & 1,51 \\
\hline
\end{tabular}

O processo de decantação é uma das formas de se clarificar este extrato. Utiliza substâncias que desestabilizam o material em suspensão e agentes floculantes que agregam estas substâncias desestabilizadas levando-as a decantar.

Para que ocorra uma adequada desestabilização (coagulação) e floculação das partículas coloidais do extrato da estévia é necessário que se encontre o valor ideal de $\mathrm{pH}$, concentração do coagulante (sulfato de alumínio) e do polímero aniônico, pois, estes fenômenos dependem da interrelação, destes fatores [3].

Os estudos referentes a este processo no que se refere ao emprego de sais inorgânicos e de agentes de floculação são escassos [2]. Por isso, objetivou-se estudar neste trabalho a clarificação do extrato cru de estévia (UEM-320) empregando o coagulante, sulfato de alumínio e o polieletrólito sintético aniônico (BETZDEARBORN ${ }^{\mathrm{TM}} \mathrm{F} 11$ ).

\section{2 - MATERIAIS E MÉTODOS}

O extrato utilizado foi obtido por infusão aquosa das folhas secas da estévia (UEM-320), a $50^{\circ} \mathrm{C}$, por cerca de 15 minutos. Os experimentos foram realizados em aparelho Jar Test Milan (modelo JT-103) em duas etapas, que se distinguiam pela quantidade do polieletrólito sintético aniônico (BETZDEARBORN $\left.{ }^{\mathrm{TM}} \mathrm{F} 11\right)$ e, na utilização do sobrenadante ou da suspensão total após o tratamento com óxido de cálcio. Este óxido além de acertar o $\mathrm{pH}$ em 8,0, 9,0 ou 10,0, também produzia uma prévia sedimentação.

Na etapa 1, o extrato de estévia teve seu pH ajustado com o óxido de cálcio, e o sobrenadante deste extrato foi tratado aplicando-se o coagulante, sulfato de alumínio, nas concentrações de 7.500, 10.000 e 12.500 ppm. Logo em seguida, foi realizada a floculação com o polímero aniônico, nas dosagens de 0,0 (controle), 2,5 e 5,0ppm.

Na etapa 2, ao extrato onde aplicou-se o óxido de cálcio, adicionou-se em seguida o coagulante, sulfato de alumínio, nas dosagens de 7.500, 10.000 e $12.500 \mathrm{ppm}$ e o polieletrólito nas dosagens de 0,0, 10,0 e 50,0ppm.

Nos testes realizados, nas duas etapas, as rotações do Jar Test foram mantidas em 120rpm por 5 segundos para a coagulação e por 1 minuto após aplicação do polímero. Em seguida, a mesma foi reduzida para $16 \mathrm{rpm}$, permanecendo assim por 30 minutos, sendo desligado automaticamente, para que se realizasse a decantação e o cálculo da velocidade decantação conforme a Equação 1 .
$V=\frac{Z}{t}$

onde:

$\mathrm{V}$ - velocidade de decantação;

$\mathrm{t}$ - tempo de decantação (15 minutos);

$Z$ - interface sólido/suspensão (12cm).

FUH \& CHIANG [5] e posteriormente MORESCHI [6] utilizaram a Equação 2 para determinações da percentagem de despigmentação, tanto para cor (absorbância em $420 \eta \mathrm{m}$ ) como para turbidez (absorbância 670ๆm.) do extrato de estévia.

$\% D=\left[1-\left(\frac{(\text { Abs } \lambda) \text { depois }}{(\text { Abs } \lambda) \text { antes }}\right)\right] x 100$

onde:

(Abs $\lambda$ )antes - valor da absorbância do extrato cru;

(Abs $\lambda$ )depois - valor da absorbância do extrato clarificado.

\section{3 - RESULTADOS E DISCUSSÃO}

Os resultados obtidos e apresentados nas Figuras 1 a 3 são referentes aos testes realizados na etapa 1 . Observa-se na Figura 1 que, usando a concentração de 7.500ppm de sulfato de alumínio e nivel 9,0 de pH ocorreu o maior valor de remoção de pigmentos. A aplicação de polímero não promoveu aumento da remoção de cor nos extratos tratados. Em pH 8,0 não ocorreu coagulação, houve acréscimo das partículas do coagulante e do polímero, acrescentou cor, ficando os valores negativos.

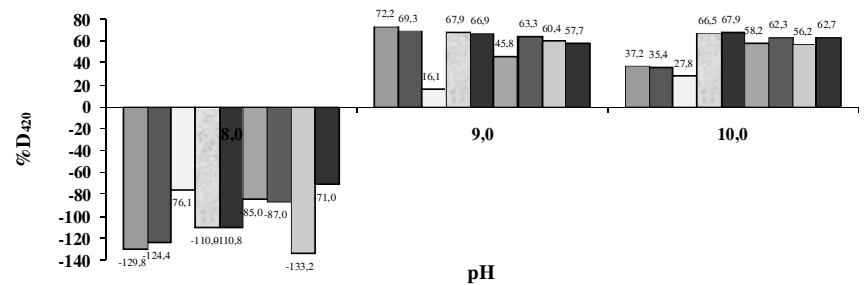

$\square 7500 ; 0,0 \square ; 7500 ; 2,5 \square 7500 ; 5,0 \square 10000 ; 0,0 \square 10000 ; 2,5 \square 10000 ; 5,0 \square 12500 ; 0,0 \square 12500 ; 2,5 \square 12500 ; 5,0$

FIGURA 1. Percentagem despigmentação 420ๆm em relação ao $\mathrm{pH}$, na etapa 1 .

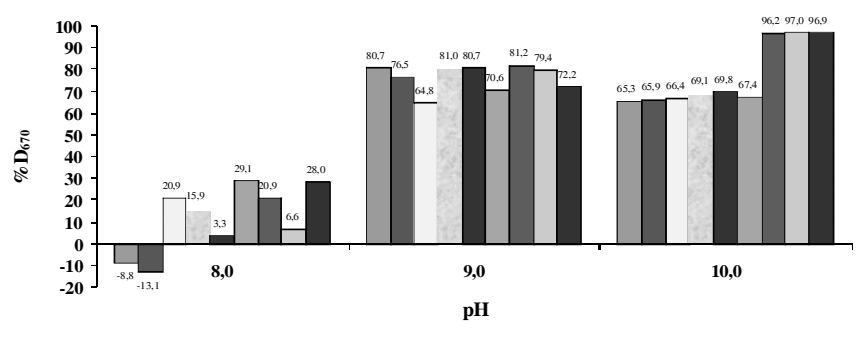

$\square ; 7500 ; 0,0 \square 7500 ; 2,5 \square 7500 ; 5,0 \backsim 10000 ; 0,0 \square 10000 ; 2,5 \square 10000 ; 5,0 \square 12500 ; 0,0 \square 12500 ; 2,5 \square 12500 ; 5,0$

FIGURA 2. Percentagem despigmentação $670 \eta \mathrm{m}$ em relação ao $\mathrm{pH}$, na etapa 1 . 
Observa-se nitidamente na Figura 2, um aumento na percentagem de despigmentação $670 \eta m$ em função do aumento do nível de $\mathrm{pH}$, dosagem do coagulante e do polieletrólito. O melhor resultado obtido para a remoção de turbidez foi na concentração de $12.500 \mathrm{ppm}$ do coagulante sulfato de alumínio, 2,5ppm do polieletrólito e em pH 10,0.

Conforme se observa na Figura 3, o aumento da velocidade de decantação ocorreu apenas para os níveis de pH 9,0 e 10,0 e como função da concentração de polieletrólito, ou seja, nos niveis de $\mathrm{pH}$ 9,0 e 10,0 a velocidade de decantação aumentou com o aumento da concentração do polímero. O maior valor conseguido foi na concentração de 5,0ppm do polímero aniônico, 12.500ppm de sulfato de alumínio e em $\mathrm{pH}$ 9,0.

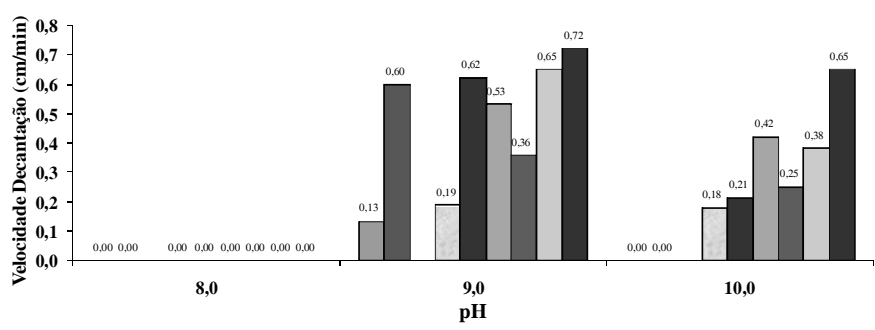

$\square 7500 ; 0,0 \square 7500 ; 2,5 \square 7500 ; 5,0 \square 10000 ; 0,0 \square 10000 ; 2,5 \square 10000 ; 5,0 \square 12500 ; 0,0 \square 12500 ; 2,5 \square 12500 ; 5,0$

FIGURA 3. Velocidade de decantação em relação ao pH, na etapa 1 .

Os resultados apresentados nas Figuras 4 a 6 são referentes aos testes realizados na etapa 2. Na Figura 4 , onde se apresentam os resultados obtidos para remoção de cor $\left(\% \mathrm{D}_{420}\right)$ verifica-se que, embora nesta etapa as concentrações de polímeros utilizadas tenham sido elevadas em relação a etapa anterior, não houve melhora na remoção de cor, pois, obteve-se como melhor resultado $72,2 \%$ na etapa 1 e $70,5 \%$ na etapa 2 , ambos em pH 9,0.

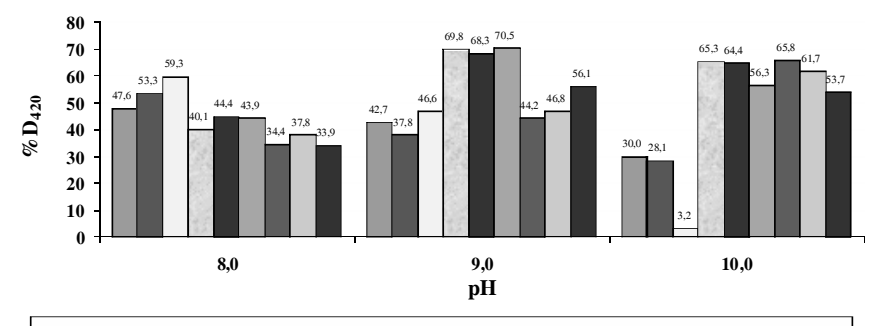

$\square 7500 ; 0.0 \square 7500 ; 10.0 \square 7500 ; 50.0 \square 10000 ; 0.0 \square 10000 ; 10.0 \square 10000 ; 50.0 \square 12500 ; 0.0 \square 12500 ; 10.0 \square 12500 ; 50.0$

FIGURA 4. Percentagem despigmentação 420ๆm em relação ao $\mathrm{pH}$, na etapa 2

Na Figura 5, observa-se que em relação a remoção de turbidez $\left(\% \mathrm{D}_{670}\right)$ para o tratamento com 7.500ppm e 10.000 ppm do coagulante, o polímero não foi fator importante, com exceção dos tratamentos realizados sob pH 10,0. Já em presença de 12.500ppm de sulfato, observa-se um aumento da percentagem de despigmentação $\left(\% \mathrm{D}_{670}\right)$ com o aumento do $\mathrm{pH}$.

Podemos verificar através da Figura 6 que, para a velocidade de decantação com aplicação do sulfato de alumínio na concentração de $7.500 \mathrm{ppm}$, o aumento da dosagem do polímero aniônico e o maior nível de $\mathrm{pH}$, determinaram os melhores resultados.

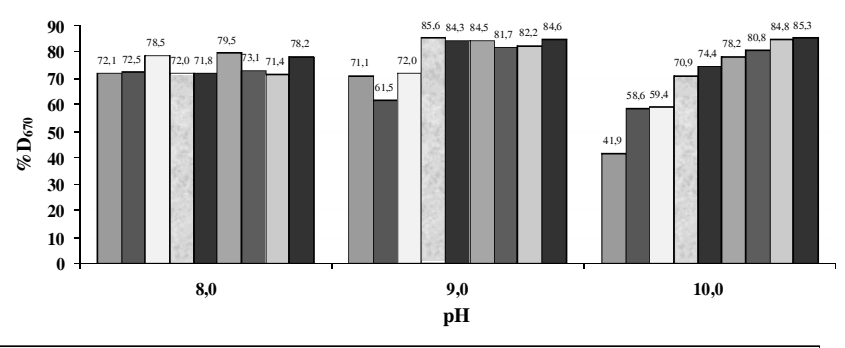

$\square 7500 ; 0.0 \square 7500 ; 10.0 \square 7500 ; 50.0 \square 10000 ; 0.0 \square 10000 ; 10.0 \square 10000 ; 50.0 \square 12500 ; 0.0 \square 12500 ; 10.0 \square 12500 ; 50.0$

FIGURA 5. Percentagem despigmentação 670ๆm em relação ao $\mathrm{pH}$, na etapa 2 .

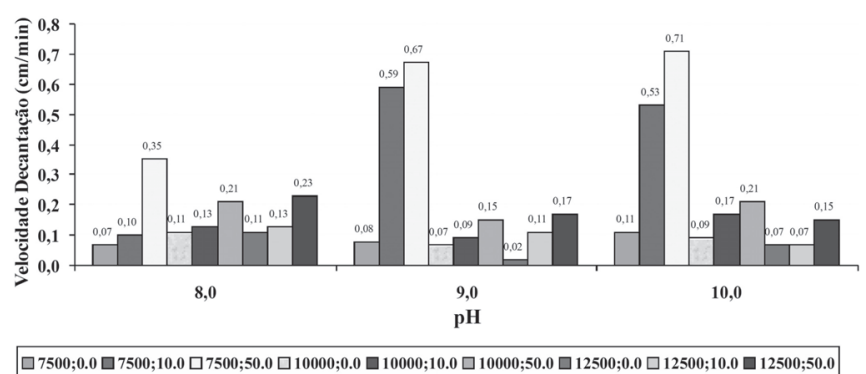

FIGURA 6. Velocidade de decantação em relação ao $\mathrm{pH}$, na etapa 2.

Nos experimentos com 10.000ppm de coagulante a concentração do polímero é um fator importante na velocidade de decantação, porém não há uma melhora com o aumento do $\mathrm{pH}$, tendo-se em vista que tanto em $\mathrm{pH}$ 8,0 quanto em 10,0 os valores e comportamento são similares.

Para a velocidade de decantação na concentração de 12.500ppm de coagulante, (sulfato de alumínio) há decréscimo com o aumento no $\mathrm{pH}$, porém a presença e/ou concentração do polímero apresenta efeitos modestos.

\section{4 - CONCLUSÕES}

- A etapa 1 dos experimentos, onde se fazia o ajuste prévio de $\mathrm{pH}$ e aplicava-se baixas concentrações de polímero, foi a que apresentou os melhores resultados, utilizando-se o óxido de cálcio e sulfato de alumínio. Para a percentagem de despigmentação em $420 \eta m$ (cor) foi obtido o valor de $72,2 \%$, na menor concentração de sulfato de alumínio (7.500ppm), em pH 9,0 e na ausência de polímero aniônico. Em 670ๆm (turbidez) a percentagem de despigmentação foi de 97,0\% nas concentrações de $12.500 \mathrm{ppm}$ de coagulante, $2,5 \mathrm{ppm}$ de polímero e $\mathrm{pH}$ 10,0.

- A velocidade de decantação obtida foi de $0,72 \mathrm{~cm} / \mathrm{min}$, em $\mathrm{pH}$ 9,0, 12.500ppm de coagulante e na dosagem de 5,0ppm para o polímero aniônico, como o melhor valor dos testes aplicados. 
- A etapa 2, que caracterizou-se por aplicação de polímero em elevadas concentrações e ajuste simultâneo de $\mathrm{pH}$, não tem aplicação recomendada, pois os valores de percentagem de despigmentação (cor e turbidez) e velocidade de decantação são menores do que os obtidos na etapa 1 , que não utilizou dosagens do polietrólito de 10,0 e 50,0ppm.

\section{5 - REFERÊNCIAS BIBLIOGRÁFICAS}

[1] BONDAREV, N.; REShetNyAK, O.; NOSOV, A. Peculiarities of Diterpenoid Steviol Glycoside Production in Vitro Cultures of Stevia rebaudiana Bertoni. Elsevier Plant Science, n. 161, p. 155-163, 2001.

[2] BUNHAK, E.J. Decantação do Extrato Aquoso de Stevia rebaudiana (Bert.) Bertoni: Avaliação do Comportamento de Agentes Floculantes. Maringá, 2002. 123p. Dissertação de Mestrado - Faculdade de Engenharia Química, Universidade Estadual de Maringá (UEM).

[3] COSTA, R.H.C. Estudo do Uso de Polímeros Naturais como Auxiliares de Floculação com Base no Diagrama de Coagulação do Sulfato de Alumínio. São Carlos, 1992. 178p. Dissertação de Mestrado - Escola de Engenharia de São Carlos, Universidade de São Paulo (USP).

[4] Fernandes, L.M. Processos de Purificação de Edulcorantes de Stevia - Estudo de Caso Projeto Stevia/UEM. PEQ/UEM, Maringá, 2001. 94p. Exame de Qualificação (Doutorado em Engenharia Química) Faculdade de Engenharia Química, Universidade Estadual de Maringá (UEM).

[5] FUH, W.S.; CHIANG, B.H. Purification of Stevioside by Membrane and Ion Exchange Process. Journal of Food Science, v. 55, n. 5, p. 1454-1457, 1990.

[6] MORESCHI, S.R.M. Purificação Extrato de Stevia rebaudiana Bertoni Através de Membranas Microporosas. PEQ/UEM, Maringá, 1999. 127p. Dissertação de Mestrado - Faculdade de Engenharia Química, Universidade Estadual de Maringá (UEM).

[7] PASgUel, A.; MEIRELES, M.A.A; MARQUES, M.O.M.; PETENATE, A.J. Extraction of Stevia Glycosides with $\mathrm{CO} 2$ + Water, $\mathrm{CO} 2$ + Ethanol, and $\mathrm{CO} 2$ + Water + Ethano. Brazilian Journal of Chemical Engineering, v. 17, n. 3, p. 271-282, 2000. 